\title{
Recurrent Thyroid Papillary Carcinoma in Children Under Ten Years Old: Report of Two Cases and Literature Review
}

\author{
Byeong-Joo Noh · Ji-Youn Sung \\ Youn-Wha Kim · Yong-Koo Park \\ Department of Pathology, Kyung Hee University \\ School of Medicine, Seoul, Korea \\ Received: January 7, 2014 \\ Revised: Febraury 18, 2014 \\ Accepted: February 28, 2014 \\ Corresponding Author \\ Yong-Koo Park, M.D. \\ Department of Pathology, Kyung Hee University \\ School of Medicine, 26 Kyungheedae-ro, \\ Dongdaemun-gu, Seoul 130-701, Korea \\ Tel: $+82-2-958-8743$ \\ Fax: +82-2-958-8730 \\ E-mail: ykpark@khmc.or.kr
}

Papillary thyroid carcinoma (PTC) in children under ten years old is very rare. To date, 18 cases of PTC in children under ten years old (including our two cases) have been reported in Korea. Here, we describe two cases of recurrent PTC with follicular variant and conventional type in an 8-yearold boy and a 7-year-old boy, respectively, and discuss clinicopathologic and molecular characteristics that differ in pediatric patients from adults.

Key Words: Thyroid cancer, papillary; Child; Recurrent
Papillary thyroid carcinoma (PTC) is extremely rare in children younger than ten years of age. The incidence of thyroid carcinoma in children and adolescents varies from 0.4 to 5.4 per 1,000,000 (0.2 to 3 in Korea). ${ }^{1,2}$ The youngest reported age at the time of diagnosis in Korea is 4.5 years. ${ }^{2}$ It is important to diagnose thyroid carcinoma earlier in children than in adults and to perform extensive whole body work ups because thyroid carcinoma tends to more frequently result in regional lymph node metastasis and distant metastasis in children than in adults. ${ }^{2}$ Although there are clinical features in children that are different from those in adults, very few molecular or clinicopathologic studies have been carried out in pediatric thyroid carcinoma. Here, we report two cases of PTC in children younger than ten years of age and discuss the clinicopathologic and molecular characteristics that differ in pediatric patients from adults.

\section{CASE REPORT}

\section{Case 1}

An asymptomatic right thyroid mass was found in an 8- year-old boy three months before the visit. He had no clinical history of exposure to radiation. Physical examination revealed multiple palpable small masses adjacent to the right thyroid nodule. Ultrasound of the neck demonstrated hypoechoic and solid nodules with inner calcification $(2.3 \times 2.0 \times 1.5 \mathrm{~cm})$ in the right thyroid lobe. Ultrasound-guided fine-needle aspiration revealed atypical cells with nuclear enlargement and pseudoinclusions. Laboratory findings of thyroid function tests were within the normal range. The patient underwent total thyroidectomy with central lymph node dissection. The pathologic diagnosis was multifocal bilateral follicular variant of PTCs with extrathyroidal extension (ETE) and regional lymph node metastasis (pT3N1) (Fig. 1). Peritumoral lymphocytic infiltration was rare. After curative surgery, he received ${ }^{131}$ I radioactive iodine therapy and T4 suppression. Four years after the initial surgery, followup ultrasound showed small nodular lesions in the previous surgical bed. Thus, the patient underwent revisional thyroidectomy followed by a third surgery, a right revisional central neck dissection. Cervical lymph node metastasis was identified. The patient has survived for two years after treatment without recurrence. 

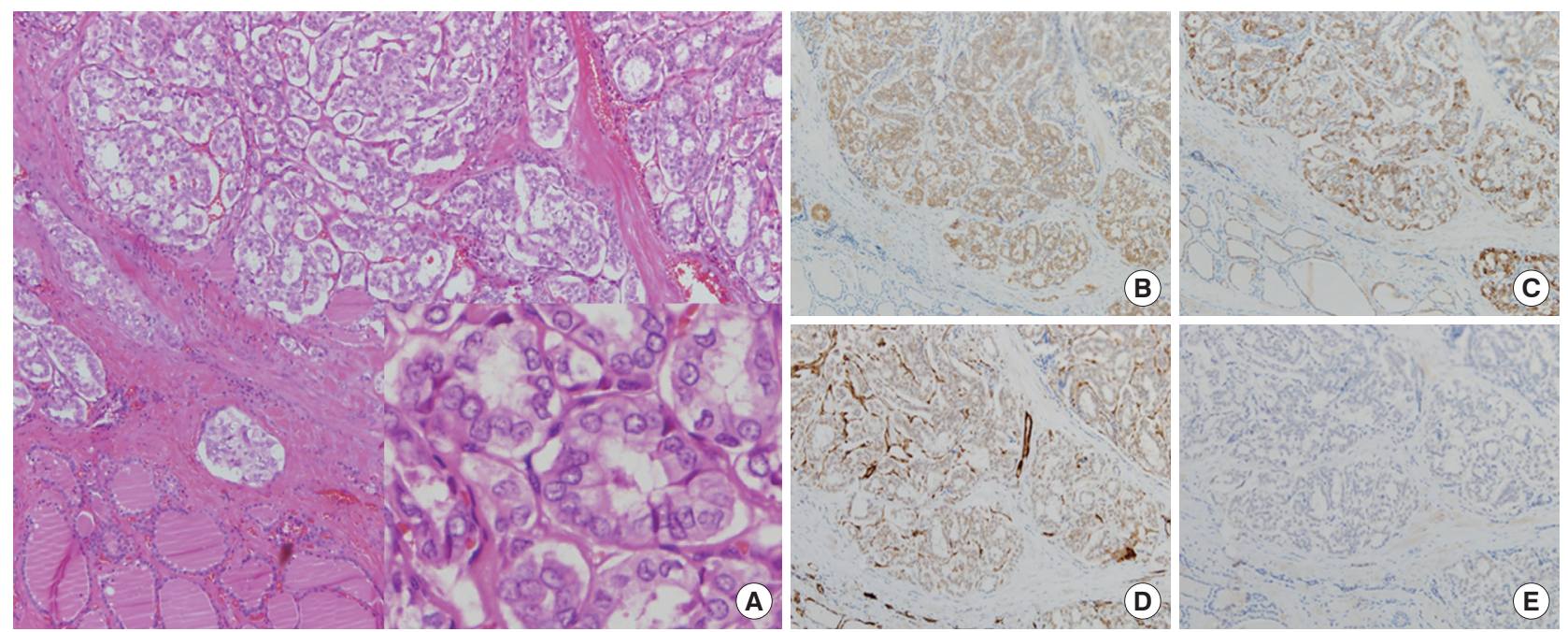

Fig. 1. Histologically, no papillary architecture is observed, and cells lining follicles show large nuclei with grooves and nuclear pseudoinclusions resembling those of conventional papillary thyroid carcinoma ( $A$, inset). Immunohistochemical staining shows positive immunoreactivity for galectin-3 (B) and cytokeratin 19 (C) and negative results for p27 (D) and cyclin D1 (E).
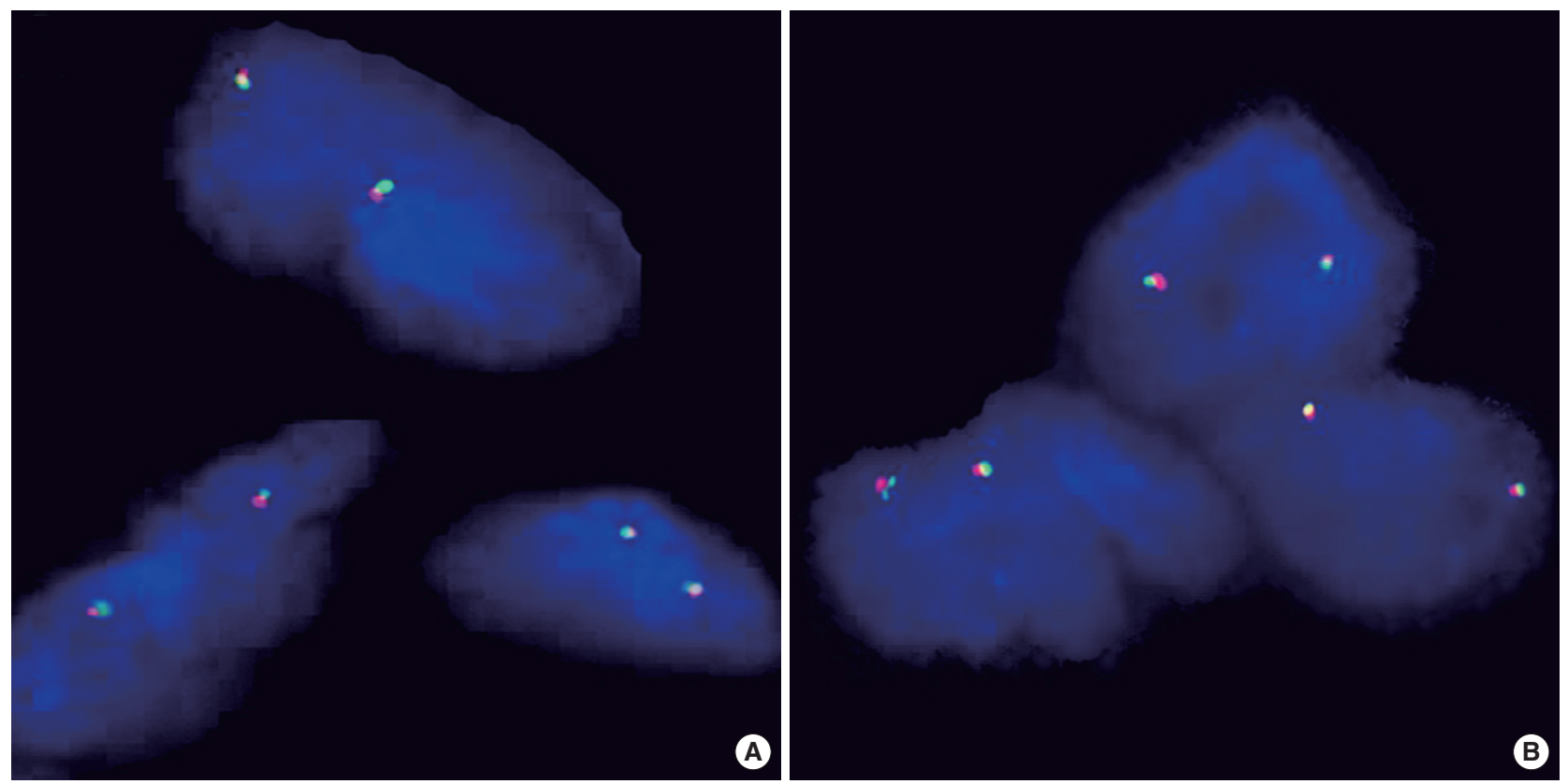

Fig. 2. The probe is a mixture of two direct-labeled probes hybridizing to the $10 q 11.21$ band. The orange fluorochrome direct-labeled probe hybridizes proximal to the RET gene, while the green fluorochrome direct-labeled probe hybridizes distal to that gene. The RET Dual Color Break Apart Probe shows one orange/green fusion signals in each nucleus in case no. 1 (A) and the same features in case no. 2 (B).

Immunohistochemical staining showed positive immunoreactivity for galectin-3 and cytokeratin 19, was negative for p27 and cyclin D1 (Fig. 1), and showed 10 leukocyte common antigen (LCA)-positive cells per high power field (HPF) and no Ki67-postive cells. BRAF, $k-R A S$, and EGFR mutations were not detected in mutational analyses using real-time polymerase chain reactions (qRT-PCR) (CFX96 Real-Time System, Bio-Rad
Laboratories, Hercules, CA, USA). ${ }^{3}$ No RET/PTC 1 or 3 rearrangements (inversions) were detected by fluorescence in situ hybridization (FISH) analysis using a RET dual color break apart probe (ZytoVision GmbH, Bremerhaven, Germany) (Fig. 2A).

\section{Case 2}

A 7-year-old-boy visited our medical center for the treatment 

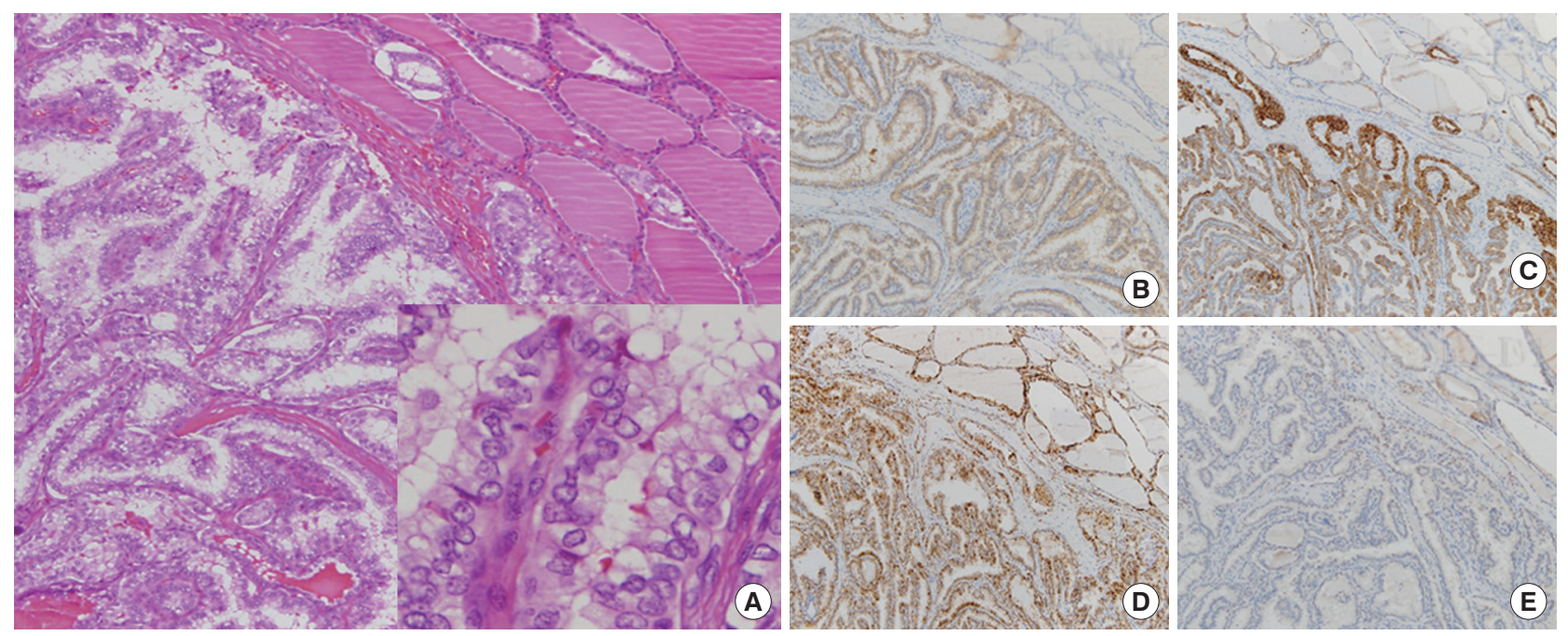

Fig. 3. Histologically, papillary architecture with enlarged or oval-shaped elongated nuclear features is observed. Nuclear clearing or ground glass appearance and irregular nuclear contours are also observed (inset, A). Immunohistochemical staining reveals positive immunoreactivity for galectin-3 (B), cytokeratin 19 (C), and p27 (D) and negative results for cyclin D1 (E).

of upper respiratory symptoms. He had no previous exposure to radiation. Ultrasound-guided fine-needle aspiration, which was carried out at other medical center, showed PTC in right thyroid lobe. Laboratory findings of thyroid function tests were within the normal range. The patient underwent total thyroidectomy with central lymph node dissection. The pathologic diagnosis was unilateral multifocal PTC with ETE and regional lymph node metastasis (pT3N1) (Fig. 3). Peritumoral lymphocytic infiltration was sparse. After the initial surgery, the patient received ${ }^{131}$ I radioactive iodine therapy and $\mathrm{T} 4$ suppression. Four years after the total thyroidectomy, we detected a recurrent right palpable lower neck mass, and the patient underwent modified radical neck dissection. Metastatic PTC was recently diagnosed in regional lymph nodes.

Immunohistochemical staining revealed positive immunoreactivity for galectin-3, cytokeratin 19, p27, was negative for cyclin D1 (Fig. 3), and showed 20 LCA-positive cells per HPF and no Ki-67-postive cells. BRAF, $k-R A S$, and EGFR genes were determined as wild-type by mutational analyses using qRT-PCR (CFX96 Real-Time System, Bio-Rad Laboratories). FISH analysis did not show any RET/PTC 1 or 3 rearrangements (inversions) using RET dual color break apart probes (ZytoVision GmbH) (Fig. 2B).

\section{DISCUSSION}

We summarized published studies of thyroid carcinomas in Korean children and adolescents and the clinicopathologic fea- tures in Table $1{ }^{2,4-10}$ Thyroid carcinoma rarely occurs in children and adolescents, but when it does, it is most common in female children. The proportion of Korean pediatric patients younger than ten years is only $11.7 \%$ (Table 1). The histologic types of thyroid cancer observed in children and adolescents are papillary (89\%) and follicular (7.8\%) carcinoma, and other types (3.2\%), exhibiting more diverse tumor types compared with those in the general population of Korean thyroid cancer patients, ${ }^{11}$ which consist primarily of papillary $(94.3 \%)$ and follicular (3.1\%) carcinoma. Additionally, the proportion of conventional PTC is much lower in young PTC patients than the proportion of other variants. The variants reported in young PTC patients include the diffuse sclerosing variant (41.2\%, predominant), conventional PTC (38.2\%), follicular (2.9\%), and cribriform-morular variants (1.5\%). ${ }^{10}$ PTC in young patients is more likely to have ETE (62.3\%), lymph node metastasis (72\%), and distant metastasis (23\%) than that in adults: adults have ETE (31.2-33\%) and lymph node metastasis $(32.1-40.9 \%) .^{12}$ The most common metastatic site is the lung $(16.2 \%)$. In view of these aggressive features at the time of diagnosis, closer and more extensive workups should be performed in young patients than in elder patients to ensure exact diagnosis and appropriate early management. ${ }^{2}$

The histopathological and immunohistochemical findings of children and adolescents with PTC are not significantly different from those of adults. However, molecular studies have suggested that there are different genetic alteration patterns in young patients. First, RET/PTC rearrangements (40\% to $70 \%$ ) 
Table 1. Clinicopathologic features of thyroid carcinoma in Korean children and adolescents

\begin{tabular}{|c|c|c|c|c|c|c|c|c|c|}
\hline No. & $\begin{array}{l}\text { No. of } \\
\text { patients }\end{array}$ & Sex & $\begin{array}{l}\text { Average age } \\
\text { (range) }\end{array}$ & $\begin{array}{c}\text { Children } \\
<10 \text {-year-old/Sex }\end{array}$ & ETE (\%) & LN metastasis (\%) & $\begin{array}{c}\text { Distant } \\
\text { metastasis (site) }\end{array}$ & Pathologic type & Reference \\
\hline 1 & 53 & $\begin{array}{l}M(9) \\
F(44)\end{array}$ & $\begin{array}{c}\text { M: } 14.2(10-19.3) \\
\text { F: } 15.1(4.5-19.7)\end{array}$ & $4 / F$ & $33(62)$ & $38(72)$ & 12 (lung) & PTC (53) & Cho et al. ${ }^{2}$ \\
\hline 2 & 11 & $\begin{array}{r}M(6) \\
F(5)\end{array}$ & $\begin{array}{l}\text { M: } 10.9(5-15) \\
\text { F: } 13.1(9-15)\end{array}$ & $1 / \mathrm{M}$ & NA & $7(64)$ & 5 (lung) & $\begin{array}{l}\text { PTC (9), FC (1), } \\
\text { HC (1) }\end{array}$ & Son et al. ${ }^{9}$ \\
\hline 3 & 68 & $\begin{array}{l}M(9) \\
F(59)\end{array}$ & $15.5 \pm 3.8(\mathrm{NA})$ & $4 / \mathrm{NA}$ & $46(68)$ & $47(69)$ & 3 (NA) & $\begin{array}{l}\text { PTC (57), FC (8), } \\
\text { PDC (3) }\end{array}$ & Koo et al. ${ }^{10}$ \\
\hline 4 & 1 & $\mathrm{~F}$ & 8 & $1 / F$ & NA & NA & 1 (lung) & PTC & Choi et al. ${ }^{8}$ \\
\hline 5 & 1 & $\mathrm{~F}$ & 13 & NA & $1(100)$ & $1(100)$ & 1 (lung) & PTC & Lee et al. ${ }^{7}$ \\
\hline 6 & 2 & $F(2)$ & $16(14-18)$ & NA & NA & $2(100)$ & 1 (lung) & PTC (2) & Lee et al. ${ }^{6}$ \\
\hline 7 & 12 & $\begin{array}{r}M(3) \\
F(9)\end{array}$ & $10.5(5.5-14)$ & 1/M, 4/F & NA & $10(83)$ & 1 (lung) & $\begin{array}{l}\text { PTC (8), FC (3), } \\
\text { mixed (1) }\end{array}$ & Choi et al..$^{5}$ \\
\hline 8 & 4 & $\begin{array}{l}M(2) \\
F(2)\end{array}$ & $10.5(9-14)$ & $1 / F$ & $1(25)$ & $3(75)$ & 1 (lung) & PTC (4) & Yang et al. ${ }^{4}$ \\
\hline Present case & 2 & M (2) & $7.5(7.2-8.1)$ & $2 / \mathrm{M}$ & $2(100)$ & $2(100)$ & 0 & PTC (2) & \\
\hline Total & 154 & $\begin{array}{l}M(31) \\
F(123)\end{array}$ & $14.4(4.5-19.7)$ & $18(11.7 \%)$ & NA & NA & 25 (16.2\%) & $\begin{array}{l}\text { PTC (137), FC (12), } \\
\text { others (5) }\end{array}$ & \\
\hline
\end{tabular}

ETE, extrathyroidal extension; LN, lymph node; M, male; F, female; PTC, papillary thyroid carcinoma; NA, not available; FC, follicular carcinoma; HC, hurthle cell carcinoma; PDC, poorly-differentiated carcinoma.

in PTC of children and adolescents are more frequently found than in PTC of adults, which are predominantly associated with BRAF mutations (45\%), ${ }^{13}$ RET/PTC rearrangements (15\%), and RAS mutations $(15 \%) .{ }^{14}$ These gene mutations and rearrangements are mutually exclusive and noncooperative. ${ }^{15}$ RET/ PTC rearrangements can activate the mitogen-activated protein kinase pathway and give rise to nuclear structures, chromatin, and cell architectures that change to papillary patterns ${ }^{16}$ in the same way as with BRAF mutations. Secondly, although $B R A F$ mutations in PTC in young adults ( $<35$ years, excluding children and adolescents) are not associated with tumor aggressiveness, RET/PTC rearrangements have been shown to be predictive of poor prognosis in PTC of young adults. ${ }^{17}$ However, to date, no previous studies have shown that the presence of RET/ PTC rearrangement is correlated with clinical outcome in children and adolescents. The correlation between genetic alterations of RET/PTC rearrangement and biological behaviors of PTC in children and adolescents should be investigated more thoroughly to clarify this relationship.

PTC in children and adolescents generally has better clinical prognosis than that in adults, although unfavorable clinical findings such as those mentioned above (multifocality, ETE, regional lymph node metastasis, and distant metastasis at the time of diagnosis) are more likely. ${ }^{18}$ Two suggestions have been presented to explain the better prognosis of PTC in children and adolescents. First, a study by Gupta $e t$ al. ${ }^{19}$ found that the proportion of lymphocytes in children is greater than in adults, suggesting that proliferating lymphocytes (tumor-associated lymphocytes) can improve disease-free survival in children and young adults. ${ }^{14}$ Secondly, pediatric patients are more susceptible to hormone therapy than adults, so that distant metastasis and relapse can be more effectively controlled by hormone therapy alone or in combination with radioiodine therapy than in adults. Because BRAF V600E mutations result in dysfunction of the sodium iodide symporter, which contributes to therapy resistance, we hypothesize that the lower incidence of $B R A F$ mutations in PTC in children and adolescents causes young patients to be more responsive to hormone therapy and radioactive iodine therapy than are adults.

We assume that various factors such as tumor-associated lymphocytes and hormonal susceptibility as well as genetic alterations might influence clinical outcome in PTC of children and adolescents. Thus, a few proliferating tumor-associated lymphocytes can explain the unfavorable clinical prognosis in our current cases, despite the lack of genetic alterations.

In conclusion, we report two cases of recurrent PTCs, follicular variant and conventional type, with no genetic alterations (BRAF, RAS, EGFR, RET/PTC1 or RET/PTC3 genes) that were detected by qRT-PCR and FISH analyses. These presenting cases are extremely rare in that they occur in young male patients younger than ten years of age.

Although some studies of PTC in children and adolescents have been conducted, the pathogenesis and molecular mechanism must still be elucidated. Therefore, further studies investigating not only the prevalence and interactions of genetic alterations, but also their clinicopathologic correlations in children 
and adolescents should be conducted.

\section{Conflicts of Interest}

No potential conflict of interest relevant to this article was reported.

\section{REFERENCES}

1. Fridman MV, Savva NN, Krasko OV, et al. Clinical and pathologic features of "sporadic" papillary thyroid carcinoma registered in the years 2005 to 2008 in children and adolescents of Belarus. Thyroid 2012; 22: 1016-24.

2. Cho EY, Lee SY, Shin CH, Yang SW. Clinical characteristics of papillary thyroid carcinoma in children and adolescent. J Korean Soc Pediatr Endocrinol 2008; 13: 65-72.

3. Jeong D, Jeong $\mathrm{Y}$, Lee $\mathrm{S}$, et al. Detection of $B R A F(\mathrm{~V} 600 \mathrm{E})$ mutations in papillary thyroid carcinomas by peptide nucleic acid clamp realtime PCR: a comparison with direct sequencing. Korean J Pathol 2012; 46: 61-7.

4. Yang SW, Park SW, Lee IS, et al. Four cases of papillary thyroid cancer in childhood. J Korean Pediatr Soc 1984; 27: 282-7.

5. Choi YW, Cho MH, Lee OK, Lee MH. Twelves cases of thyroid carcinoma in children. J Korean Pediatr Soc 1986; 29: 79-85.

6. Lee JW, Moon JH, Shin JH, Lee H. Papillary cancer of the thyroid gland in childhood and adolescence: a review of 3 patients. J Korean Soc Pediatr Endocrinol 1999; 4: 237-42.

7. Lee CB, Yang SC, Park YS, et al. A case of pulmonary metastasis of thyroid papillary carcinoma which was mistaken for miliary tuberculosis. J Korean Soc Endocrinol 2000; 15: 600-5.

8. Choi EJ, Kim JR, Lee CW, et al. A case of thyroid papillary carcinoma presenting as a miliary pulmonary infiltration in 8 year old girl. Pediatr Allergy Respir Dis 2003; 13: 271-6.

9. Son CH, Ko JM, Lee JH, Choi JH, Yoo HW. Clinical course and out- come of thyroid carcinoma in childhood. J Korean Soc Pediatr Endocrinol 2006; 11: 57-63.

10. Koo JS, Hong S, Park CS. Diffuse sclerosing variant is a major subtype of papillary thyroid carcinoma in the young. Thyroid 2009; 19: 1225-31.

11. Ahn HY, Park YJ. Incidence and clinical characteristics of thyroid cancer in Korea. Korean J Med 2009; 77: 537-42.

12. Kim WJ, Bae MJ, Yi YS, et al. Clinicopathologic characteristics of papillary microcarcinoma in the elderly. J Korean Thyroid Assoc 2013; 6: 69-74.

13. Kim WY, Ko YS, Hwang TS, et al. A case of multifocal papillary thyroid carcinoma consisting of one encapsulated follicular variant with BRAF K601E mutation and three conventional types with BRAF V600E mutation. Korean J Pathol 2013; 47: 293-8.

14. Bhaijee F, Nikiforov YE. Molecular analysis of thyroid tumors. Endocr Pathol 2011; 22: 126-33.

15. Soares $\mathrm{P}$, Trovisco V, Rocha AS, et al. BRAF mutations and RET/ $P T C$ rearrangements are alternative events in the etiopathogenesis of PTC. Oncogene 2003; 22: 4578-80.

16. Fischer AH, Bond JA, Taysavang P, Battles OE, Wynford-Thomas D. Papillary thyroid carcinoma oncogene (RET/PTC) alters the nuclear envelope and chromatin structure. Am J Pathol 1998; 153: 1443-50.

17. Sassolas G, Hafdi-Nejjari Z, Ferraro A, et al. Oncogenic alterations in papillary thyroid cancers of young patients. Thyroid 2012; 22: 1726.

18. Xing M. BRAF mutation in papillary thyroid cancer: pathogenic role, molecular bases, and clinical implications. Endocr Rev 2007; 28: 742-62.

19. Gupta S, Patel A, Folstad A, et al. Infiltration of differentiated thyroid carcinoma by proliferating lymphocytes is associated with improved disease-free survival for children and young adults. J Clin Endocrinol Metab 2001; 86: 1346-54. 